\title{
Decreased argininosuccinate synthetase expression in Thai patients with cholangiocarcinoma and the effects of ADI-PEG20 treatment in CCA cell lines
}

\author{
SOMPHON ROEKSOMTAWIN ${ }^{1,2}$, PANIDA NAVASUMRIT ${ }^{1-3}$, SOMCHAMAI WARAPRASIT ${ }^{1}$, \\ VARABHORN PARNLOB ${ }^{1}$, THANIYA SRICHARUNRAT ${ }^{4}$, VAJARABHONGSA BHUDHISAWASDI ${ }^{5,6}$, \\ NIRAMOL SAVARAJ ${ }^{7}$ and MATHUROS RUCHIRAWAT ${ }^{1-3}$
}

\begin{abstract}
${ }^{1}$ Laboratory of Environmental Toxicology, Chulabhorn Research Institute; ${ }^{2}$ Chulabhorn Graduate Institute, Bangkok 10210; ${ }^{3}$ Center of Excellence on Environmental Health and Toxicology, CHE, Ministry of Education, Bangkok 10300; ${ }^{4}$ Chulabhorn Hospital, Bangkok 10210; ${ }^{5}$ Department of Surgery, Faculty of Medicine, Khon Kaen University, Khon Kean 40000; ${ }^{6}$ Laboratory of Chemical Carcinogenesis, Chulabhorn Research Institute, Bangkok 10210 , Thailand; ${ }^{7}$ Department of Medicine, University of Miami, Miller School of Medicine, Miami, FL 33125, USA
\end{abstract}

Received September 20, 2017; Accepted May 3, 2018

DOI: $10.3892 / 01.2018 .8807$

\begin{abstract}
Cholangiocarcinoma (CCA) is a severe cancer with poor prognosis. The aim of the present study was to explore the expression of argininosuccinate synthetase (ASS), as well as the possibility of using pegylated arginine deiminase (ADI-PEG20) for the treatment of CCA. ASS expression was determined in CCA specimens from 40 patients in Thailand. Immunohistochemical detection of ASS and determination of the proliferative index, Ki-67, were carried out in paraffin-embedded sections of these specimens, as well as in two CCA cell lines, HuCCA and RmCCA-1, derived from CCA samples from patients in Thailand. In total, $\sim 45 \%$ of the CCA specimens had low ASS expression, and the level of expression was significantly negatively associated with cell differentiation $(\mathrm{P}<0.05)$ and $\mathrm{Ki}-67$ expression $(\mathrm{P}<0.05)$. The level of ASS expression in tumor cells was significantly lower than that in non-tumor cells $(1.3$-fold, $\mathrm{P}<0.05)$. The HuCCA cell line had significantly lower levels $(\mathrm{P}<0.05)$ of ASS expression at the mRNA and protein levels relative to those of normal human immortalized fibroblast cells (BJ-1). By contrast, the RmCCA-1 cell line showed no significant difference. In addition, the effects of ADI-PEG20 on growth
\end{abstract}

Correspondence to: Professor Mathuros Ruchirawat, Laboratory of Environmental Toxicology, Chulabhorn Research Institute, 54 Kamphaeng Phet 6 Road, Laksi, Bangkok 10210, Thailand E-mail: mathuros@cri.or.th

Professor Niramol Savaraj, Department of Medicine, University of Miami, Miller School of Medicine, Rm 2B127, VA Medical Center, 1201 NW 16th Street, Miami, FL 33125, USA

E-mail:nsavaraj@med.miami.edu

Key words: cholangiocarcinoma, argininosuccinate synthetase, $\mathrm{Ki}-67$, pegylated arginine deiminase, arginine deprivation inhibition, apoptosis and cell cycle arrest were determined in HuCCA and RmCCA-1 cells. ADI-PEG20 treatment reduced cell viability and cell proliferation in the two CCA cell lines, though it had no effect in immortalized BJ-1 cells. Furthermore, ADI-PEG20 treatment significantly increased G0/G1 cell cycle arrest in HuCCA, though not in RmCCA-1 cells. ASS silencing in the RmCCA-1 cell line significantly enhanced its sensitivity to ADI-PEG20 treatment. Results from the in vitro study demonstrated that ADI-PEG20 has antitumor activity against CCA with low ASS expression.

\section{Introduction}

Liver cancer is the fifth and seventh most common malignancy in men and women, respectively. Approximately $85 \%$ of all cases occur in developing countries (1). Cholangiocarcinoma (CCA) is the second most common type of liver cancer. It accounts for $15 \%$ of all primary liver cancers, and $3 \%$ of all gastrointestinal tumors worldwide $(2,3)$. There are two types of CCA. Intrahepatic CCA is highly malignant and its incidence is increasing worldwide. Extrahepatic CCA is less malignant and more stable in incidence (4). While CCA is uncommon in the United States and Europe, it is very common in Thailand and other Southeast Asian countries. The overall incidence of CCA in the USA is 2.9 cases $/ 100,000$ patients (4), while in the North and Northeast regions of Thailand it is 30.9 cases/100,000 patients (5). Liver fluke (Opisthorchis viverrini) is a well-established contributory factor to CCA in Thailand. Chronic inflammation induced by liver fluke infection causes malignant transformation of the bile duct (6). The primary treatment for CCA is surgical resection. However, patients often present at a late stage, which makes surgical resection much more difficult. Despite this difficulty, $25 \%$ of these cases undergo the procedure successfully $(5,7)$. The 5 -year patient survival rates are $20-32 \%$ for intrahepatic CCA and 30-42\% for extrahepatic CCA (8). This difference is most likely due to microscopic metastatic lesions 
and the frequency of positive surgical margins (9). Despite extensive research and various new treatment modalities, such as immunotherapy or signal transduction inhibitors that have been developed to treat tumors, these have not been effective in CCA treatment. Thus, other novel modalities of treatment are needed. In this study, we have explored a new direction for treating CCA by targeting the metabolic vulnerability of the disease.

In intrahepatic CCA, tumor cells proliferate rapidly (10) and thus require significant amounts of amino acids, such as arginine, to support their growth. Advanced malignancies, including: hepatocellular carcinoma (HCC), melanoma, prostate cancer, pancreatic cancer, mesothelioma, renal cell carcinoma, sarcoma, and small cell lung cancer, are deficient in argininosuccinate synthetase (ASS) expression, the rate-limiting enzyme in arginine synthesis (11). Generally, arginine is synthesized from citrulline through two-step reactions catalyzed by ASS and argininosuccinate lyase (12). Tumor cells that have low ASS expression are unable to synthesize arginine and must depend on extracellular arginine (13). Thus, arginine becomes an essential amino acid. Depletion of arginine in the extracellular environment can be achieved by the arginine deiminase (ADI) enzyme (14), a bacterial enzyme which degrades arginine to citrulline. Pegylated ADI has been created by conjugating ADI with polyethylene glycol (ADI-PEG20) to improve its half-life and to suppress its immunogenicity. Treatment of arginine auxotrophic cancers with ADI-PEG20 can result in tumor cell death due to lack of ASS $(11,15)$. Several phase I/II clinical trials have demonstrated promising clinical benefits with low toxicity when using ADI-PEG20 to treat patients with ASS-deficient cancers, such as HCC, metastatic melanoma, and mesothelioma (16). A Phase III clinical trial was undertaken to treat HCC patients with ADI-PEG20 (11). As reported at ASCO 2016, the randomized trial showed no overall survival benefit linked to ADI-PEG20 treatment compared to placebo; however, some patients appeared to benefit from the treatment.

This study aimed to investigate the expression of ASS in intrahepatic CCA, and to explore whether ADI-PEG20 could be useful for CCA treatment. We determined the levels of ASS expression at both the mRNA and protein level and assessed cell proliferation via $\mathrm{Ki}-67$ expression in CCA specimens from Thai patients. We also used two CCA cell lines derived from Thai patients as in vitro models to test the inhibitory effect of ADI-PEG20 and correlate with ASS expression. Silencing of ASS expression was also carried to further confirm that ASS expression is a key determinant for the antitumor effect of ADI-PEG20.

\section{Materials and methods}

Patients and tissue samples. A total of 40 CCA patients, comprising of 24 males and 16 females with a median age of 60 years (range 48-73 years) was recruited for this study. All cases underwent surgical resection. The clinicopathological features of the patients were collected including gender, age, type of CCA, histopathological differentiation, TNM staging, lymphovascular invasion, perineural invasion and viral hepatitis status. Information regarding liver fluke infection was obtained from questionnaires. Only 2 cases were reported.
No specific test for liver fluke infection was performed. Paraffin-embedded tissues representing 40 CCA patients, 38 of which were intrahepatic CCA and 2 of which were perihilar CCA cases, were obtained from Chulabhorn hospital, and from Srinagarind hospital, which is affiliated to Khon Kaen Medical University. The histological types of the CCA tissues were classified according to the World Health Organization classification (17). This study was conducted according to the Helsinki declaration for international health research, and was approved by the Human Research Ethics Committee of Chulabhorn Research Institute, Bangkok, Thailand (project no. 013/2559 on 17 August 2016). All the subjects gave written informed consent for participation prior to enrollment in the study.

Cell culture and treatment. Two human CCA cell lines (RmCCA-1 and HuCCA) and a human fibroblast cell line (BJ-1) were used in this study. The RmCCA-1 and HuCCA cell lines were established from intrahepatic CCA specimens derived from Thai patients. The characterization of these two cell lines has previously been published $(18,19)$. These CCA cell lines were maintained in DMEM media supplemented with $10 \%$ FBS and Penicillin/Streptomycin. The cells were obtained from the Chulabhorn Research Institute, Thailand. BJ-1 cells were obtained from the ATCC. The BJ-1 cells were maintained on EMEM supplemented with 10\% FBS and Penicillin/Streptomycin.

For ADI-PEG20 treatment, cells were seeded and allowed to attach overnight at $37^{\circ} \mathrm{C}$, then treated for 3 days with $0.1 \mu \mathrm{g} / \mathrm{ml}$ of ADI-PEG20 (kindly provided by Polaris Pharmaceuticals Inc., San Diego, CA, USA). Controls did not receive ADI-PEG20.

For treatment with arginine-free medium, the medium was prepared as described in Savaraj et al (20) with minor modifications. Briefly, the medium was pretreated with $0.1 \mu \mathrm{g} / \mathrm{ml}$ of ADI-PEG20 for 3 days prior to use.

Where ASS siRNA treatment is indicated, cells were pretreated with $50 \mathrm{nM}$ of either pooled non-target scramble control siRNA (siNT) or 3 unique 27 mer siRNA obtained from OriGene Technologies, Inc. (Rockville, MD, USA; cat. no. SR300322). The transfection was accomplished using INTERFERin (Polyplus-transfection, New York, USA) according to the manufacturer's protocol. After 3 days of siRNA transfection with/without ADI treatment, cells were harvested and assayed for ASS expression by western blot, and for study of growth inhibition or apoptotic effect of ADI-PEG20 treatment.

Immunohistochemistry. The ASS expression level and Ki-67 proliferation index were determined for paraffin-embedded CCA tissues (3- $\mu \mathrm{m}$ sections). Sections were de-paraffinized in xylene and washed sequentially with $100 \%$ and $95 \%$ ethanol. Endogenous peroxidase activity was blocked by incubating slides in 3\% hydrogen peroxide for $20 \mathrm{~min}$ at room temperature. Antigen retrieval was carried out with target retrieval solution (Dako; Agilent Technologies, Inc., Santa Clara, CA, USA) for $20 \mathrm{~min}$ at $90^{\circ} \mathrm{C}$, followed by the blocking of non-specific binding with the biotin blocking system (Dako; Agilent Technologies, Inc.) for $10 \mathrm{~min}$. The primary antibodies used were anti-ASS (mouse monoclonal, 1:100 dilution; 
BD Biosciences, Franklin Lakes, NJ, USA) and anti-Ki-67 (MIB-1, mouse monoclonal, 1:100 dilution; Dako; Agilent Technologies, Inc.). Sections were incubated with primary antibodies overnight at room temperature in a moist chamber. Subsequently, the sections were washed 3 times in PBS and incubated with biotinylated anti-mouse immunoglobulins (Dako; Agilent Technologies, Inc.) for $15 \mathrm{~min}$, followed by incubation with streptavidin peroxidase conjugate (Dako; Agilent Technologies, Inc.) for $15 \mathrm{~min}$. The color reaction was developed with Liquid DAB Substrate-Chromogen (1:50 v/v; Dako; Agilent Technologies, Inc.). The sections were counterstained with hematoxylin, dehydrated with graded ethanol and mounted with Cytoseal-60. For the negative controls, the primary antibody was replaced with antibody diluent at the appropriate dilution.

Slides were viewed by a pathologist using an Axio Imager Z2 Microscope (Zeiss GmbH, Jena, Germany) equipped with a HXG40c Baumer digital camera and the HistoFAXS ${ }^{\circledR}$ Version 4.1 (TissueGnostics GmbH, Vienna, Austria) image analysis system. In the same tissue sections, the tumor areas were selected and identified from the invasive areas, whereas the surrounding non-tumor areas were selected from those in the vicinity of the tumor areas but with apparent normal cells. At least 4 different regions of tumor and non-tumor areas were selected for each specimen.

ASS expression in CCA specimens was classified as low or high according to a previous report (21). Briefly, IHC analysis of ASS and Ki-67 expression in CCA specimens was determined by HistoFAXS software Version 4.1 and viewed at 100x magnification. The measurement parameters included mean intensity and percentage of stained cells. For ASS expression, the mean intensity of ASS in tumor areas was normalized to the mean intensity of ASS expression in non-tumor areas from the same specimen. The mean ASS expression ratio (tumor/non-tumor areas) from 40 patients was 0.77 , where levels below or above 0.77 were classified as low- or high-ASS expression, respectively. For Ki-67 expression in each specimen, the mean intensity and percentage of stained cells was determined from at least 4 different regions of tumor areas.

In situ immunocytochemistry. All cell lines were seeded and allowed to attach overnight at $37^{\circ} \mathrm{C}$ on chamber slides. After treatment for 3 consecutive days, cells were fixed on a permeabilized membrane by incubation with $95 \%$ ethanol for $10 \mathrm{~min}$, followed by incubation in antibody diluent (Dako; Agilent Technologies, Inc.) for $10 \mathrm{~min}$. The immunocytochemical detection of ASS and Ki-67 was performed as described for immunohistochemical detection.

Western blot analysis. Cells for total cell lysate production were harvested using a cell-scraper in cold PBS. Cell were lysed with RIPA buffer (Cell Signaling Technology, Inc., Danvers, MA, USA) plus protease inhibitor cocktail (EMD Millipore, Billerica, MA, USA), passed several times through a 23G needle, and centrifuged as previously described (14). For each cell lysate, the protein concentration was determined using the Bio-Rad Protein Assay (Bio-Rad Laboratories, Inc., Hercules, CA, USA), according to the manufacturer's protocol. Twenty micrograms of protein was electrophoresed on $12 \%$ polyacrylamide gels containing sodium dodecyl sulfate and transferred to a nitrocellulose membranes by electro-blotting (GE Healthcare Life Sciences, Little Chalfont, UK). The membrane was then washed and incubated with monoclonal antibodies against ASS (1:1,000; BD Biosciences). Detection of ASS expression was done using an ECL Western Blotting Detection kit (GE Healthcare Life Sciences), according to the manufacturer's instructions. Expression was normalized to internal controls, using a mouse antibody against $\beta$-actin (Sigma-Aldrich; Merck KGaA, Darmstadt, Germany).

Gene expression analysis by reverse transcription-quantitative polymerase chain reaction ( $R T-q P C R)$. Total RNA was extracted from the cells using a PerfectPure RNA Cultured Cell kit (5 PRIME, Hamburg, Germany) according to the manufacturer's instructions. One-step RT-qPCR was performed with a LightCycler 480 (Roche Diagnostics GmbH, Mannheim, Germany). Briefly, RNA (20 ng) was mixed with 1X Quantitect SYBR Green RT-PCR Master Mix (Qiagen $\mathrm{GmbH}$, Hilden, Germany), $0.2 \mu \mathrm{l}$ QuantiTect RT mix (Qiagen $\mathrm{GmbH}$ ) and ASS primers (forward: 5'-GAGGATGCCTGA ATTCTACA-3' and reverse: 5'-GTTGGTCACCTTCAC AGG-3') in a total volume of $20 \mu \mathrm{l}$. The RT-qPCR conditions were as follows: $50^{\circ} \mathrm{C}$ for $20 \mathrm{~min}, 95^{\circ} \mathrm{C}$ for $15 \mathrm{~min}$ followed by 45 cycles of: $95^{\circ} \mathrm{C}$ for $15 \mathrm{sec}, 58^{\circ} \mathrm{C}$ for $20 \mathrm{sec}$ and $72^{\circ} \mathrm{C}$ for $30 \mathrm{sec}$. Expression of $A S S$ was quantified using the $\Delta \Delta \mathrm{Cq}$ method and normalized to GAPDH (forward primer: 5'-TCT TCCAGGAGCGAGATCC-3' and reverse primer: 5'-TTGTCA TGGATGACCTTGGC-3').

Growth inhibitory assay. Cell viability was determined by 3-(4,5-dimethylthiazol-2yl)-2,5-diphenyl tetrazolium bromide (MTT) assay. Cells treated with ADI-PEG20 $(0.05-1 \mu \mathrm{g} / \mathrm{ml})$ were incubated with $20 \mu \mathrm{l} \mathrm{MTT}\left(5 \mathrm{mg} / \mathrm{ml}\right.$ in PBS) at $37^{\circ} \mathrm{C}$ for $4 \mathrm{~h}$. The medium was then removed and $200 \mu \mathrm{l}$ dimethylsulfoxide (DMSO) was added to each well followed by incubation for $5 \mathrm{~min}$ at room temperature. Viability was assessed using a microplate reader (SoftMax Pro; Molecular Devices, Sunnyvale, CA, USA) at $570 \mathrm{~nm}$. Relative cell viability was determined and expressed as percentage of control.

Apoptosis assay. The percentage of cells undergoing apoptosis after ADI-PEG20 treatment was determined using the Muse ${ }^{\mathrm{TM}}$ Annexin V and Dead Cell kit (EMD Millipore). Treated and control cells were collected in medium containing $1 \% \mathrm{FBS}$, mixed with the Muse Annexin V and Dead Cell Reagent and incubated in the dark at room temperature for 30 min according to the kit instructions. The percentage of apoptotic cells was determined using a Muse Cell Analyzer (EMD Millipore).

Cell cycle analysis. Cell cycle arrest was determined using the Muse ${ }^{\mathrm{TM}}$ Cell Cycle kit (EMD Millipore), according to the manufacturer's instructions. Briefly, control and treated cells were fixed with ice-cold $70 \%$ ethanol at $-20{ }^{\circ} \mathrm{C}$ for $3 \mathrm{~h}$, washed with PBS and stained with PI/RNAse reagent for $30 \mathrm{~min}$. The percentage of cells in each phase of the cell cycle was analyzed using a Muse Cell Analyzer (EMD Millipore).

Statistical analysis. Data were expressed as the mean $\pm \mathrm{SE}$. All statistical analyses were performed using the Stata software package (version 10.0; StataCorp LP, College Station, 
TX, USA). A paired t-test was used to compare the immunohistochemistry data for ASS expression between non-tumor and tumor cells. The Chi-square test was used to determine differences between categorical variables. For the in vitro study, the Student's t-test was used to compare the two sets of data and analysis of variance was used to compare $>3$ sets of data using the Bonferroni test for post hoc analysis. Pearson's correlation was used to assess correlation between proliferation and expression level of ASS or CCA stage.

\section{Results}

Immunohistochemical analysis of ASS expression in CCA specimens and its relationship to clinicopathological parameters. In order to investigate whether CCA may be an arginine auxotrophic cancer, the levels of ASS expression in 40 human CCA specimens were determined using immunohistochemistry. Our results showed that the intensity of cytoplasmic ASS staining in cancer cells was much lower than in the surrounding non-tumor liver cells in specimens from CCA patients (Fig. 1A-C). The level of ASS expression, quantified as the relative intensity of ASS staining, is summarized in Table I. In CCA, the mean intensity of ASS staining in tumor cells was 1.3-fold lower than for non-tumor cells (33.56 vs. 44.23, $\mathrm{P}<0.05)$. The mean of the ASS expression ratio (tumor/non-tumor) in CCA was 0.77 . In an attempt to assess the association of ASS expression and clinicopathological features of CCA, the level of ASS expression stratified by the level below or above the mean intensity ratio was arbitrarily categorized as low- or high-ASS expressed groups, respectively (a ratio above 0.77 was categorized as high; a ratio lower than 0.77 was categorized as low). As shown in Table II, approximately $45 \%$ of the CCA samples were classified as the low ASS-expressed group. It is interesting to note that the level of ASS expression showed a significant association with cell differentiation in CCA $(\mathrm{P}=0.030)$ (Table II), and was also associated with poor pathological features of CCA (Fig. 1D-F). However, no association between the level of ASS expression and gender, age, viral hepatitis status, lymphovascular invasion, lymph node metastasis, or TNM stage was observed. These results suggest that a reduction of ASS expression could potentially be a molecular marker for potential CCA treatment by arginine deprivation.

Histologic correlation of Ki-67 expression in CCA. The Ki-67 proliferative index is widely used as a prognostic marker in many types of cancers. To assess the associations between $\mathrm{Ki}-67$ and the histopathological parameters of CCA, immunohistochemical staining for Ki-67 was performed in 40 human CCA specimens. As shown in Fig. 2A, the mean intensities of Ki-67 staining for stages 2, 3, and 4 were 49.97, 55.52 and 62.69, respectively. An increased expression of Ki-67 was positively correlated with advanced stage CCA specimens $(\mathrm{r}=0.678, \mathrm{P}<0.05$; Fig. 2A). In line with the mean intensity of $\mathrm{Ki}-67$, the mean percentage of $\mathrm{Ki}-67$ stained cells was also positively correlated with advanced stage CCA specimens $(\mathrm{r}=0.781, \mathrm{P}<0.05)$ as shown in Fig. 2B. In addition, the ratio of ASS expression was negatively correlated $(\mathrm{P}<0.05)$ with the mean level of Ki-67 intensity ( $r=-0.395$; Fig. 2C) and percentage of stained cells ( $r=-0.346$; Fig. 2D).
Table I. Immunohistochemical detection of ASS expression in CCA specimens derived from Thai patients.

Level of ASS expression

CCA specimens (Arbitrary unit)

Tumor tissue

$33.56 \pm 2.44^{\mathrm{a}}$

Surrounding non-tumor tissue

$32.57(3.99-87.87)$

Ratio (tumor/non-tumor)

$44.23 \pm 2.27$

42.89 (22.69-86.58)

$0.77 \pm 0.04$

$0.84(0.11-1.23)$

The values are expressed as the mean \pm standard error, and as the median (min-max). ${ }^{\text {a }}<0.05$ vs. surrounding non-tumor tissue. CCA, cholangiocarcinoma; ASS, argininosuccinate synthetase.
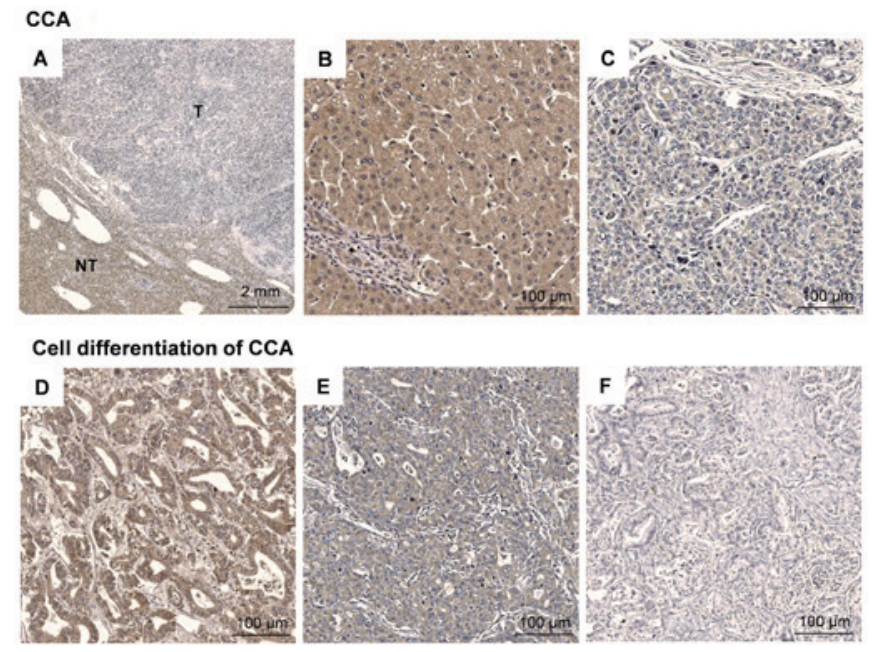

Figure 1. Immunohistochemical detection of ASS expression in paraffin-embedded sections. Paraffin-embedded sections of samples from 40 CCA patients were subjected to immunohistochemical detection of ASS expression. The cytoplasmic staining of ASS was performed using 3,3'-diaminobenzidine in CCA specimens. (A) The intensity of ASS staining in tumor cells (indicated by $\mathrm{T}$ ) was decreased when compared with non-tumor (normal) cells (indicated by NT). The enlarged images (magnification, $\mathrm{x} 100$ ) of stained (B) non-tumor (ASS expression=44.23) and (C) tumor cells (ASS expression=33.56) are also presented. Levels of ASS expression in cells that are (D) well, (E) moderately and (F) poorly differentiated. The ASS expression was highest in well-differentiated CCA (ASS expression=37.56) followed by moderately-differentiated CCA (ASS expression=33.47) and poorly-differentiated CCA (ASS expression=28.70). Scale bars, $100 \mu \mathrm{m}$. CCA, cholangiocarcinoma; ASS, argininosuccinate synthetase.

In vitro study to characterize the level of ASS expression in human CCA cell lines and their responses to ADI-PEG20 treatment

Level of ASS expression in human CCA cell lines. Basal expression of ASS in RmCCA-1 and HuCCA cells was determined at both the protein and mRNA levels in relation to that in non-cancer cells (BJ-1). As shown in Fig. 3A, immunoblot detection of the ASS protein in various cell lines showed that relative ASS protein expression was significantly lower in HuCCA cells $(0.61$-fold, $\mathrm{P}<0.05)$, but slightly higher in RmCCA-1 cells (1.04-fold, $\mathrm{P}>0.05)$, compared to that in BJ-1 cells. The cytoplasmic staining of the ASS protein by in situ 
Table II. Association between the level of ASS and clinicopathological features of CCA.

\begin{tabular}{|c|c|c|c|c|}
\hline \multirow[b]{2}{*}{ Variable } & \multirow[b]{2}{*}{ Patients (n) } & \multicolumn{2}{|c|}{ ASS expression ratio ${ }^{a}$} & \multirow[b]{2}{*}{ P-value } \\
\hline & & Low (n) & $\operatorname{High}(\mathrm{n})$ & \\
\hline All cases & 40 & 18 & 22 & - \\
\hline Sex & & & & 0.154 \\
\hline Male & 24 & 13 & 11 & \\
\hline Female & 16 & 5 & 11 & \\
\hline Age (years) & & & & 0.057 \\
\hline$<60$ & 20 & 12 & 8 & \\
\hline$\geq 60$ & 20 & 6 & 14 & \\
\hline Viral status (Hepatitis B or C) & & & & 0.949 \\
\hline Yes & 18 & 8 & 10 & \\
\hline No & 22 & 10 & 12 & \\
\hline Cell differentiation & & & & 0.030 \\
\hline Well & 22 & 8 & 14 & \\
\hline Moderately & 13 & 5 & 8 & \\
\hline Poorly & 5 & 5 & 0 & \\
\hline Lymphovascular invasion & & & & 0.900 \\
\hline Yes & 33 & 15 & 18 & \\
\hline No & 7 & 3 & 4 & \\
\hline Lymph node metastasis & & & & 0.775 \\
\hline Yes & 19 & 9 & 10 & \\
\hline No & 21 & 9 & 12 & \\
\hline TNM stage & & & & 0.673 \\
\hline I-II & 3 & 1 & 2 & \\
\hline III-IV & 37 & 17 & 20 & \\
\hline
\end{tabular}

${ }^{a}$ The mean of ASS expression ratio (0.77) was used to separate the cases into 2 groups; Low or high ASS expressed-groups, which have ASS expression ratio below or above the mean, respectively. CCA, cholangiocarcinoma; ASS, argininosuccinate synthetase; TNM, tumor-node-metastasis.

immunocytochemistry in all cell lines showed the same trend as that determined by immunoblotting (RmCCA-1> BJ-1> HuCCA) (Fig. 3B). In addition, the expression levels of the ASS protein in all cell lines correlated with the level of ASS mRNA (Fig. 3C).

Effect of ADI-PEG20 treatment on cell viability and cell proliferation of human CCA cell lines. In order to investigate whether there is an association between the expression of ASS and sensitivity to arginine deprivation caused by ADI-PEG20 treatment, the dose-response to ADI-PEG20-induced growth inhibition in CCA cell lines was determined. ADI-PEG20 treatment significantly inhibited growth of RmCCA-1 and HuCCA cells by decreasing both the percentage of viable cells (Fig. 4) and the proliferative activity as determined by Ki-67 expression (Fig. 5). As shown in Fig. 4, a statistically significant decrease in cell viability in both RmCCA-1 and HuCCA cells after ADI-PEG20 treatment was observed in a dose-dependent manner. However, only a slight decrease in cell viability was found in normal immortalized cells (BJ-1). Subsequent to ADI-PEG20 treatment, a significant reduction in Ki-67 expression was observed in HuCCA (1.3- and 1.4-fold at
0.1 and $1.0 \mu \mathrm{g} / \mathrm{ml}$, respectively) and RmCCA-1 cells (1.2-fold at $1.0 \mu \mathrm{g} / \mathrm{ml})(\mathrm{P}<0.05$; Fig. 5A). A significant reduction in the percentage of $\mathrm{Ki}-67$ stained cells $(\mathrm{P}<0.05)$ was found in ADI-PEG20-treated HuCCA (2- and 2.2-fold at 0.1 and $1.0 \mu \mathrm{g} / \mathrm{ml}$, respectively) and RMCCA-1 (1.4-fold at $1.0 \mu \mathrm{g} / \mathrm{ml})$ cells as shown in Fig. 5B. As expected, these results suggested that the HuCCA cell line, which has low ASS expression, is more sensitive to ADI-PEG20 treatment. On the other hand, ADI-PEG20 treatment had no effect on BJ-1 cells, even though the BJ-1 cell line expressed ASS at a lower level than RmCCA-1 cells. This is not surprising, since tumor cells grow rapidly and require more arginine than normal immortalized cells. Intracellular arginine supply through the urea cycle is inadequate to support rapid proliferation; therefore, $\mathrm{RmCCA}-1$ cells grow more slowly and have lower Ki-67 levels.

Effect of ADI-PEG20 treatment on cell cycle arrest and apoptosis in human CCA cell lines. We further determined whether growth inhibition resulting from ADI-PEG20 was the consequence of cell cycle arrest and/or apoptotic cell death. Cell cycle analysis was performed using propidium iodide staining, which corresponds to the DNA content of the cells. 

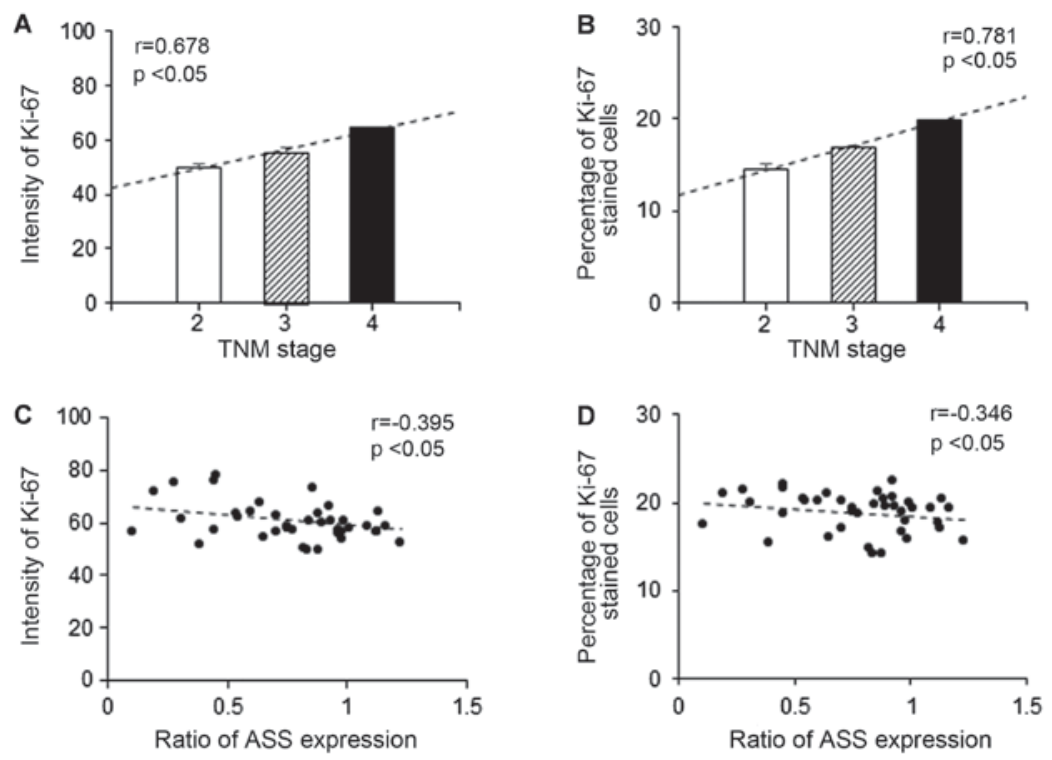

Figure 2. Expression of Ki-67 in CCA specimens stratified by TNM stage and its correlation with ASS expression. Paraffin-embedded sections of samples from CCA patients were subjected to immunohistochemical detection of proliferative activity by using the Ki-67 labeling index. The correlations are shown between: The intensity of Ki-67 and (A) the TNM stage of CCA in patients, and (C) ASS expression, as well as between the percentage of Ki-67 stained cells and (B) the TNM stage of CCA in patients and (D) ASS expression. CCA, cholangiocarcinoma; ASS, argininosuccinate synthetase; TNM, tumor-node-metastasis.
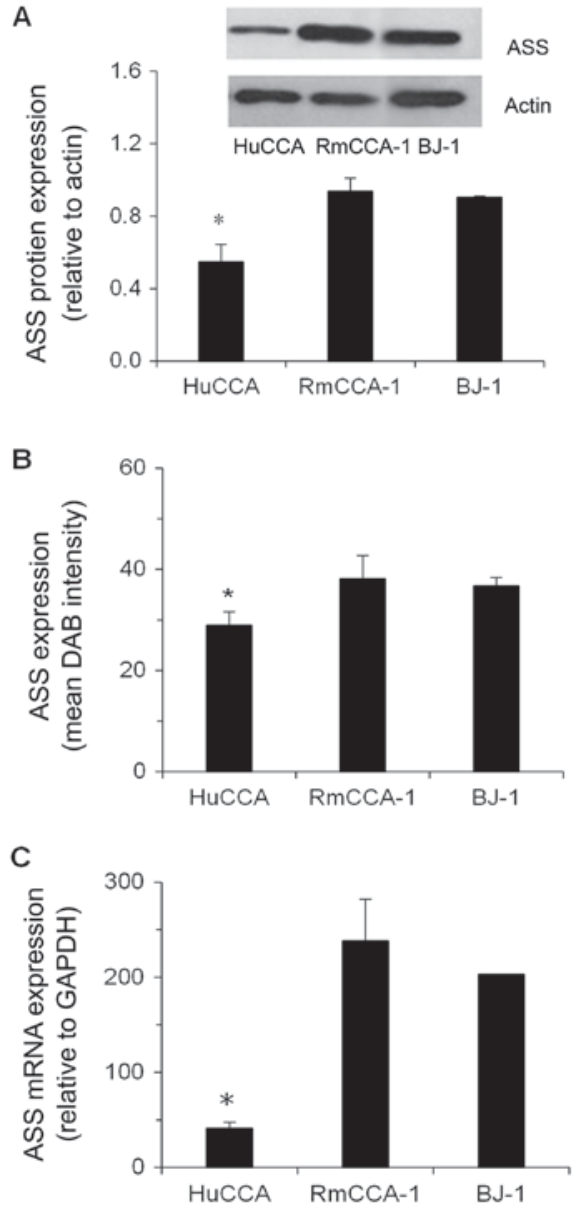

Figure 3. Level of ASS expression in human CCA cell lines. ASS protein levels in HuCCA, RmCCA-1 and BJ-1 cells were determined by (A) western immunoblotting and (B) in situ immunocytochemistry. (C) The expression of ASS mRNA was determined by reverse transcription-quantitative polymerase chain reaction. For western blot analysis, the expression of actin was used as the loading control. Data are presented as the mean \pm standard error of three independent experiments. " $\mathrm{P}<0.05$ vs. BJ-1 cells. CCA, cholangiocarcinoma; ASS, argininosuccinate synthetase; DAB, 3,3'-diaminobenzidine.
As shown in Fig. 6A, treatment with ADI-PEG20 in HuCCA cells significantly decreased population of cells in the $S$ and G2/M phases, while it increased accumulation of cells in the G0/G1 phase, suggesting G0/G1 arrest in HuCCA cells treated with ADI-PEG20. However, such effect was not observed in RmCCA-1 cells treated with ADI-PEG20 (Fig. 6B).

The induction of apoptotic cell death by ADI-PEG20 was assessed using Annexin V staining, which is an apoptosis marker. As shown in Fig. 6C, an increase in apoptotic cell population was observed in HuCCA cells treated with ADI-PEG20 treatment, whereas RmCCA-1 cells displayed only a slight increase in the number of apoptotic cells upon ADI-PEG20 treatment. The results showed that HuCCA cells, which have low ASS expression, are more sensitive to ADI-PEG20 treatment when compared to RmCCA-1 cells, which have a higher level of ASS expression. These results suggest that the efficacy of ADI-PEG20 treatment in CCA cells depends on the level of ASS expression.

Effect of ADI-PEG20 treatment on ASS knockdown RmCCA-1 cells. To further confirm that the levels of ASS expression is an important contributory factor to ADI-PEG20 sensitivity, we knocked down ASS expression in RmCCA-1 cells using siRNA and determined the sensitivity to ADI-PEG20 in these transfectants. As shown in Fig. 7B, ADI-PEG20 treatment decreased the viability of RmCCA- $1^{\text {siAss }}$ cells (20\% reduction) with no effect on the viability of RmCCA-1 cells treated with pooled non-target scramble control siRNA (RmCCA-1 ${ }^{\text {sinT }}$ cells). This data suggests that silencing of ASS increases sensitivity of these cells to ADI-PEG20 treatment.

Results of a fluorescence-activated cell sorting analysis of cellular apoptosis on ADI-PEG20-treated HuCCA, RmCCA-1, RmCCA-1 $1^{\text {sinT }}$ and RmCCA-1 $1^{\text {siAss }}$ cells are shown in Fig. 7C. Treatment with ADI-PEG20 did not significantly induce apoptosis in either the HuCCA or RmCCA-1 and RmCCA ${ }^{\text {sinT }}$ cell lines, while using arginine-free medium slightly increased the 


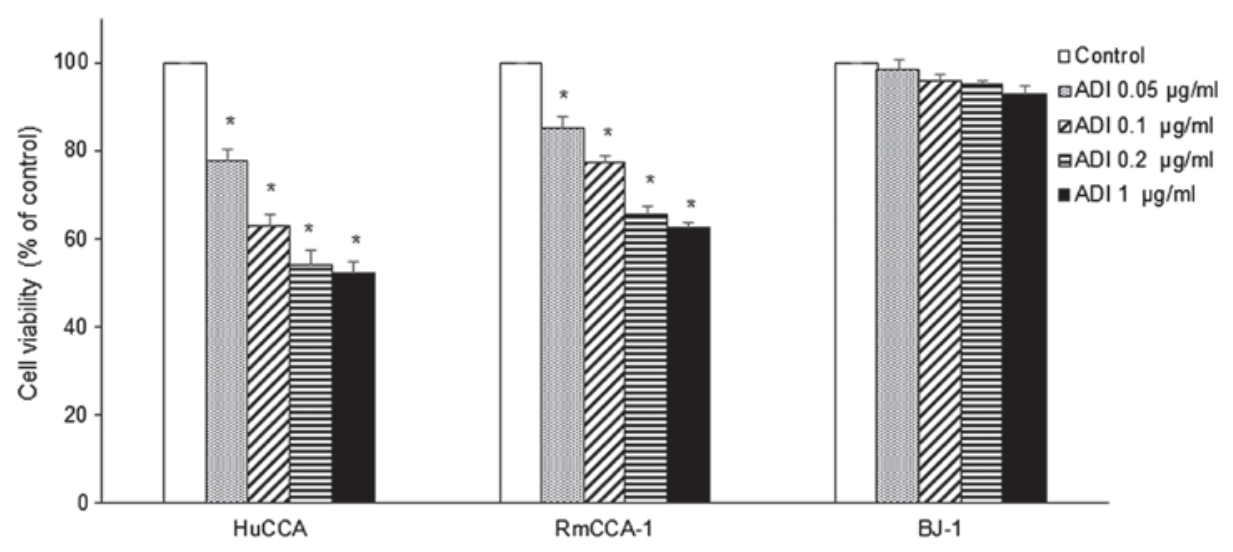

Figure 4. Effects of ADI-PEG20 treatment on cell death in human CCA cell lines. HuCCA, RmCCA-1 and BJ-1 cells were treated with ADI-PEG20 $(0.05-1 \mu \mathrm{g} / \mathrm{ml})$ for 3 days. The percentage of viable cells was determined by MTT assay. Data are presented as the mean \pm standard error of three independent experiments. ${ }^{*} \mathrm{P}<0.05$ vs. control. ADI-PEG20, pegylated arginine deiminase.
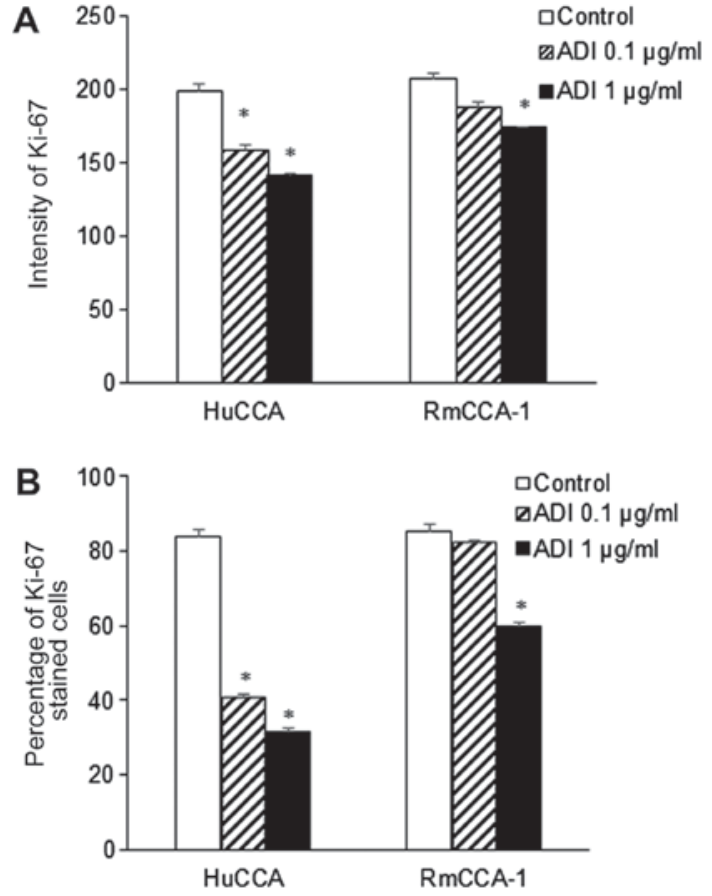

Figure 5. Effect of ADI-PEG20 treatment on Ki-67 expression in human CCA cell lines. HuCCA and RmCCA-1 cells were treated with 0.1 or $1 \mu \mathrm{g} / \mathrm{ml}$ ADI-PEG20 for 3 days. The proliferation activity was determined using the Ki-67 labelling index. In situ immunocytochemical detection was used to determine Ki-67 intensity and the percentage of Ki-67 stained cells. Histogram plots present the (A) intensity of Ki-67 and (B) percentage of Ki-67 stained cells in either the control or ADI-PEG20-treated HuCCA and RmCCA-1 cells. The data are presented as the mean \pm standard error of three independent experiments. ${ }^{*} \mathrm{P}<0.05$ vs. control. CCA, cholangiocarcinoma; ADI-PEG20, pegylated arginine deiminase.

percentage of apoptotic cells in both HuCCA and RmCCA-1 cells, as well as its non-target transfectants. However, treatment with ADI-PEG20 significantly induced apoptosis in RmCCA- $1^{\text {siASs }}$ cells (25\%) while treatment with arginine-free medium increased the apoptotic cells to $45 \%$. The increase in cell death using arginine-free medium is most likely due to the fact that the arginine-free duration was longer, while treatment with ADI-PEG20 results in a gradual loss of arginine. Taken together, our results strongly suggest that ADI-PEG20 treatment could be a potential therapy for CCA patients with low ASS expression.

\section{Discussion}

Depleting arginine to halt tumor growth was first exploited to treat cancer a decade ago, but the approach has never made it to clinical trials due to the lack of a suitable enzyme to degrade arginine (16). In the past few years, ADI-PEG20, a new generation of pegylated ADI, has entered into clinical trials. Antitumor response following administration of ADI-PEG20 has been observed primarily in tumors with low or no ASS expression $(11,22)$. However, re-expression of the enzyme has been shown, both in vitro and in vivo, to contribute to ADI-PEG20 resistance, especially in melanoma (23-25). ASS expression is known to be positively regulated by c-Myc and negatively regulated by HIF-1 $\alpha$ (25). Epigenetic regulation is also important depending on tumor type (23).

At present, there is no data on ASS expression in CCA. In this study, we demonstrated that $45 \%$ of CCA samples showed relatively low ASS protein expression in tumor cells. Low levels of ASS expression were significantly associated with unfavorable histopathologic differentiation, but not with gender, age, viral hepatitis, lymphovascular invasion, lymph node metastasis or TNM stage. Similar to this finding, it has been shown that in human pancreatic cancer (26), HCC, myxofibrosarcoma and bladder cancer, decreased ASS expression correlated with histopathological grading (27). For instance, a 1.29-fold reduction of ASS expression in tumor cells of HCC specimens was associated with poor tumor differentiation (21). However, there was no association found between ASS expression and histopathological characteristics of tumors in a cohort study of lung cancer patients (27). The differences among the reports could be due to the different methods used for quantitation. In our study, we quantitated the ASS expression in tumor cells by comparing it with that in the adjacent normal cells in the same slides, thus, avoiding the variations created by staining in different batches.

$\mathrm{Ki}-67$ has been used as a proliferative indicator in many types of cancers, such as breast cancer, lymphoma and renal carcinoma as well as CCA $(28,29)$. Upregulation of Ki-67 has been shown to be associated with poorly differentiated 
A HuCCA

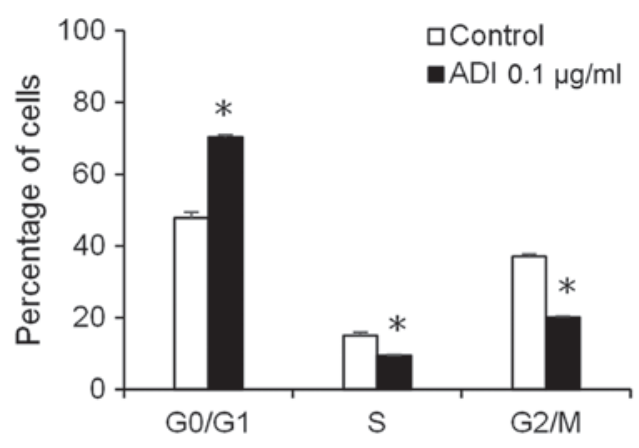

B RmCCA-1

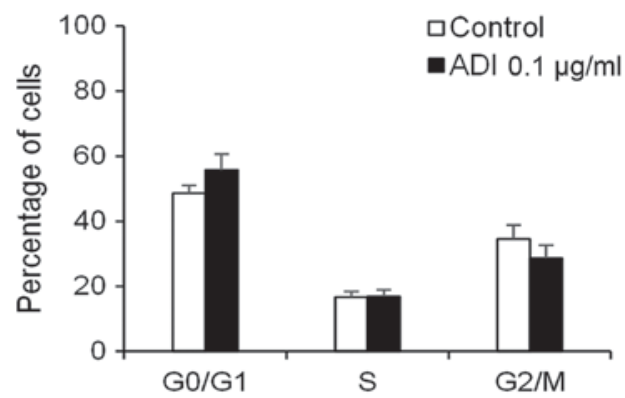

C

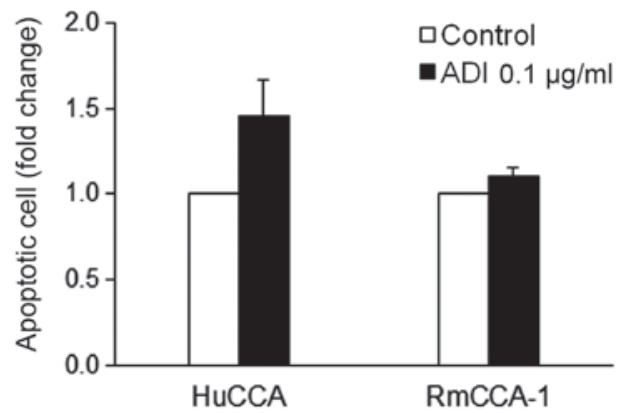

Figure 6. Effect of ADI-PEG20 treatment on cell cycle arrest and induction of apoptosis in human CCA cell lines. HuCCA and RmCCA-1 cells were treated with $0.1 \mu \mathrm{g} / \mathrm{ml}$ ADI-PEG20 for 3 days. Fluorescence-activated cell sorting analysis was performed to determine the percentage of cells in each phase of the cell cycle (G0/G1, S and G2/M phase) in (A) HuCCA and (B) RmCCA-1 cells, and (C) the number of apoptotic cells in HuCCA and RmCCA-1 cells. The data are presented as the mean \pm standard error of three independent experiments. " $\mathrm{P}<0.05$ vs. control. CCA, cholangiocarcinoma; ADI-PEG20, pegylated arginine deiminase.

tumors, advanced stage disease, poor prognosis, and decreased survival rates in CCA patients $(28,30,31)$. In line with previous findings, our study showed a significant association between increased expression of Ki-67, either as intensity or percentage of Ki-67 stained cells, in addition to advanced stage disease in CCA samples. Additionally, in CCA samples the level of ASS expression was found to be inversely correlated with the proliferative activity, as determined by Ki-67 expression. Overall, low ASS expression could be associated with poorer clinicohistopathological findings and a higher Ki-67 proliferative index in CCA samples $(32,33)$.

In summary, this is the first report to show a variation in ASS expression in CCA. In $45 \%$ of CCA cases, low ASS expression was associated with poorly differentiated cells and more aggressive tumors $(33,34)$. In addition, the
A
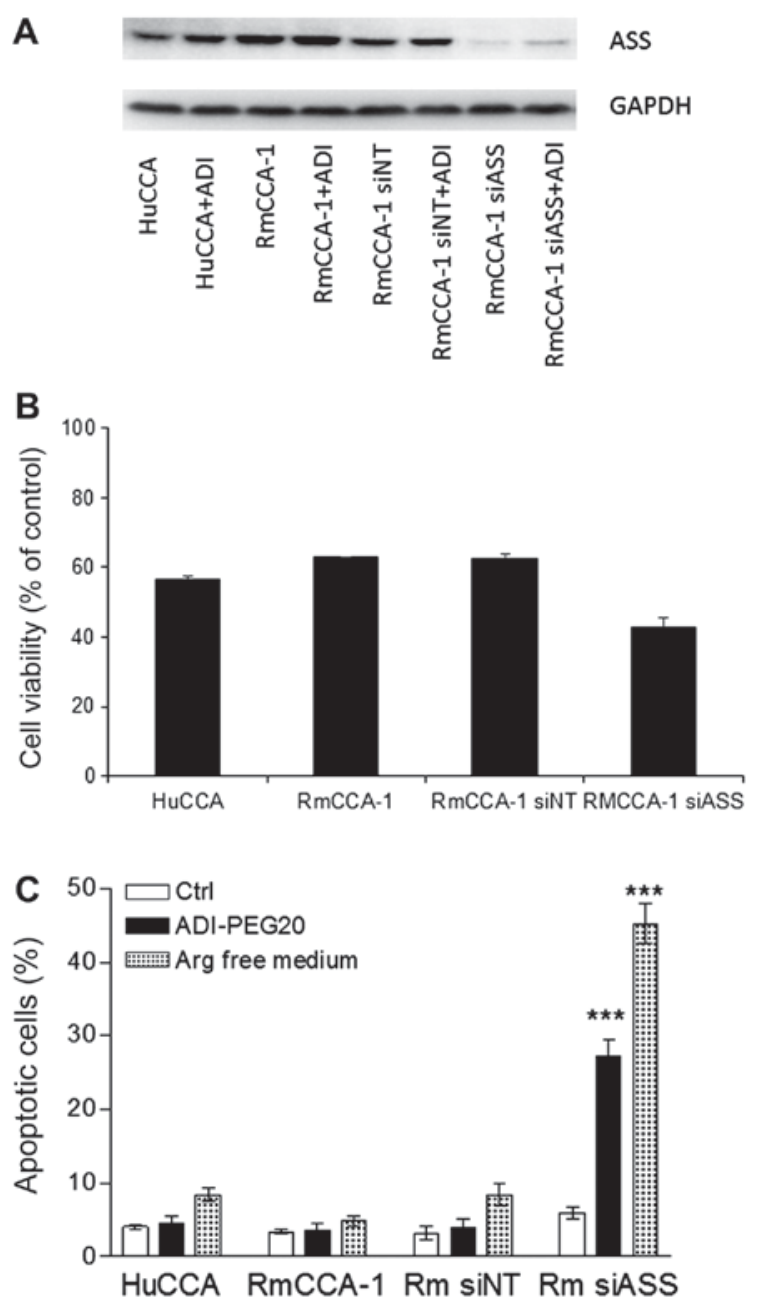

Figure 7. Effect of arginine deprivation using ADI-PEG20 treatment or arginine-free medium in ASS knockdown human CCA cell lines. (A) Immunoblot analysis of ASS protein levels in HuCCA, RmCCA-1, RmCCA- $1^{\text {sinT }}$ and RmCCA- $1^{\text {siASS }}$ with or without treatment with $0.1 \mu \mathrm{g} / \mathrm{ml}$ ADI-PEG20 for 3 days. (B) The growth inhibitory effect of ADI-PEG20 using MTT assay in HuCCA, RmCCA-1, RmCCA- ${ }^{\text {siNT }}$ and RmCCA- $1^{\text {siAss }}$ cells. (C) The percentage of apoptotic cells as determined by fluorescence-activated cell sorting analysis following treatment with ADI-PEG20 for 3 days or culturing in arginine-free medium for 3 days in HuCCA, RmCCA-1, RmCCA-1 $1^{\text {sinT }}$ and RmCCA-1 $1^{\text {siASS }}$ cells. The data are presented as the mean \pm standard error of three independent experiments. ${ }^{* * *} \mathrm{P}<0.001$ vs. control. CCA, cholangiocarcinoma; ASS, argininosuccinate synthetase; ADI-PEG20/ADI, pegylated arginine deiminase; si-, small interfering; NT, non-target scramble control.

in vitro study confirmed that low ASS expression in CCA cells was important for the efficacy of ADI-PEG20 treatment of CCA. This group of CCA patients with low ASS expression may be amenable to treatment with arginine deprivation. Even though this treatment may not eliminate the tumor cells, it will halt their proliferation. In other tumor types, a combination of ADI-PEG20 with chemotherapeutic agents has been shown to improve its therapeutic efficacy with no overt toxicity (35). Thus, combination treatments employing ADI-PEG20 with chemotherapeutic agents that have shown some activity against CCA, such as gemcitabine or cisplatin/oxaliplatin, may produce a higher response rate and improve overall survival in patients with this disease (35-37). 


\section{Acknowledgements}

The authors would like to thank Polaris Pharmaceuticals Inc. (San Diego, CA, USA) for providing ADI-PEG20 for the present study.

\section{Funding}

The present study was supported by The Chulabhorn Research Institute and The Center of Excellence on Environmental Health and Toxicology (Bangkok, Thailand) as well as a partial support from VA Merit Review Award (1BX003328) to Dr. Niramol Savaraj.

\section{Availability of data and materials}

The datasets used and/or analyzed during the current study are available from the corresponding author on reasonable request.

\section{Authors' contributions}

SR performed analysis of western immunoblotting of ASS, cell cycle arrest and apoptosis in CCA cell lines. PN conducted the data analysis and manuscript preparation. SW and VP were responsible for the analysis of mRNA expression and IHC detection of ASS, respectively. TS and VB contributed to preparation of tissue slides from CCA specimens and histopathological evaluation. NS contributed to the study design, specifically silencing of ASS expression using siRNA. MR, as the principal investigator, conceived and designed the study and experiments, sought funding support and served a key role in developing the manuscript. All authors read and approved the final manuscript.

\section{Ethics approval and consent to participate}

The present study was approved by The Human Research Ethics Committee of Chulabhorn Research Institute (Bangkok, Thailand; project no. 013/2559 on 17th August 2016). All of the subjects gave written informed consent for participation prior to enrollment in the study.

\section{Consent for publication}

Written informed consent was obtained from all participants.

\section{Competing interests}

The authors declare that they have no competing interests.

\section{References}

1. Ferlay J, Shin HR, Bray F, Forman D, Mathers C and Parkin DM: Estimates of worldwide burden of cancer in 2008: GLOBOCAN 2008. Int J Cancer 127: 2893-2917, 2010.

2. Aljiffry M, Abdulelah A, Walsh M, Peltekian K, Alwayn I and Molinari M: Evidence-based approach to cholangiocarcinoma: A systematic review of the current literature. J Am Coll Surg 208: 134-147, 2009.

3. Parkin DM, Ohshima H, Srivatanakul P and Vatanasapt V: Cholangiocarcinoma: Epidemiology, mechanisms of carcinogenesis and prevention. Cancer Epidemiol Biomarkers Prev 2: 537-544, 1993
4. Mosadeghi S, Liu B, Bhuket T and Wong RJ: Sex-specific and race/ethnicity-specific disparities in cholangiocarcinoma incidence and prevalence in the USA: An updated analysis of the 2000-2011 surveillance, epidemiology and end results registry. Hepatol Res 46: 669-677, 2016.

5. Butthongkomvong K, Sirachainan E, Jhankumpha S, Kumdang S and Sukhontharot OU: Treatment outcome of palliative chemotherapy in inoperable cholangiocarcinoma in Thailand. Asian Pac J Cancer Prev 14: 3565-3568, 2013.

6. Smout MJ, Sotillo J, Laha T, Papatpremsiri A, Rinaldi G, Pimenta RN, Chan LY, Johnson MS, Turnbull L, Whitchurch CB, et al: Carcinogenic parasite secretes growth factor that accelerates wound healing and potentially promotes neoplasia. PLoS Pathog 11: e1005209, 2015.

7. Friman S: Cholangiocarcinoma-current treatment options. Scand J Surg 100: 30-34, 2011.

8. Murakami Y, Uemura K, Sudo T, Hashimoto Y, Nakashima A, Kondo N, Sakabe R, Ohge H and Sueda T: Prognostic factors after surgical resection for intrahepatic, hilar, and distal cholangiocarcinoma. Ann Surg Oncol 18: 651-658, 2011.

9. Patel T: Cholangiocarcinoma-controversies and challenges. Nat Rev Gastroenterol Hepatol 8: 189-200, 2011.

10. Andersen JB and Thorgeirsson SS: Genetic profiling of intrahepatic cholangiocarcinoma. Curr Opin Gastroenterol 28: 266-272, 2012.

11. McAlpine JA, Lu HT, Wu KC, Knowles SK and Thomson JA: Down-regulation of argininosuccinate synthetase is associated with cisplatin resistance in hepatocellular carcinoma cell lines: Implications for PEGylated arginine deiminase combination therapy. BMC Cancer 14: 621, 2014.

12. Bowles TL, Kim R, Galante J, Parsons CM, Virudachalam S, Kung HJ and Bold RJ: Pancreatic cancer cell lines deficient in argininosuccinate synthetase are sensitive to arginine deprivation by arginine deiminase. Int J Cancer 123: 1950-1955, 2008.

13. Delage B, Fennell DA, Nicholson L, McNeish I, Lemoine NR, Crook T and Szlosarek PW: Arginine deprivation and argininosuccinate synthetase expression in the treatment of cancer. Int $\mathrm{J}$ Cancer 126: 2762-2772, 2010.

14. Savaraj N, Wu C, Kuo MT, You M, Wangpaichitr M, Robles C, Spector S and Feun L: The relationship of arginine deprivation, argininosuccinate synthetase and cell death in melanoma. Drug Target Insights 2: 119-128, 2007.

15. Savaraj N, You M, Wu C, Wangpaichitr M, Kuo MT and Feun LG: Arginine deprivation, autophagy, apoptosis (AAA) for the treatment of melanoma. Curr Mol Med 10: 405-412, 2010.

16. Phillips MM, Sheaff MT and Szlosarek PW: Targeting arginine-dependent cancers with arginine-degrading enzymes: Opportunities and challenges. Cancer Res Treat 45: 251-262, 2013.

17. Hahnvajanawong C, Chaiyagool J, Seubwai W, Bhudhisawasdi V, Namwat N, Khuntikeo N, Sripa B, Pugkhem A and Tassaneeyakul W: Orotate phosphoribosyl transferase mRNA expression and the response of cholangiocarcinoma to 5-fluorouracil. World J Gastroenterol 18: 3955-3961, 2012.

18. Rattanasinganchan $\mathrm{P}$, Leelawat K, Treepongkaruna SA, Tocharoentanaphol C, Subwongcharoen S, Suthiphongchai T and Tohtong R: Establishment and characterization of a cholangiocarcinoma cell line (RMCCA-1) from a Thai patient. World J Gastroenterol 12: 6500-6506, 2006.

19. Sirisinha S, Tengchaisri T, Boonpucknavig S, Prempracha N, Ratanarapee S and Pausawasdi A: Establishment and characterization of a cholangiocarcinoma cell line from a Thai patient with intrahepatic bile duct cancer. Asian Pac J Allergy Immunol 9: 153-157, 1991.

20. Savaraj N, Wu C, Li YY, Wangpaichitr M, You M, Bomalaski J, He W, Kuo MT and Feun LG: Targeting argininosuccinate synthetase negative melanomas using combination of arginine degrading enzyme and cisplatin. Oncotarget 6: 6295-6309, 2015.

21. Thongkum A, Wu C, Li YY, Wangpaichitr M, Navasumrit P, Parnlob V, Sricharunrat T, Bhudhisawasdi V, Ruchirawat M and Savaraj N: The combination of arginine deprivation and 5-fluorouracil improves therapeutic efficacy in argininosuccinate synthetase negative hepatocellular carcinoma. Int J Mol Sci 18: pii: E1175, 2017.

22. Beddowes E, Spicer J, Chan PY, Khadeir R, Corbacho JG, Repana D, Steele JP, Schmid P, Szyszko T, Cook G, et al: Phase 1 dose-escalation study of pegylated arginine deiminase, cisplatin, and pemetrexed in patients with argininosuccinate synthetase 1-deficient thoracic cancers. J Clin Oncol 35: 1778-1785, 2017. 
23. Locke M, Ghazaly E, Freitas MO, Mitsinga M, Lattanzio L, Lo Nigro C, Nagano A, Wang J, Chelala C, Szlosarek P and Martin SA: Inhibition of the polyamine synthesis pathway is synthetically lethal with loss of argininosuccinate synthase 1 . Cell Rep 16: 1604-1613, 2016.

24. Long Y, Tsai WB, Wangpaichitr M, Tsukamoto T, Savaraj N, Feun LG and Kuo MT: Arginine deiminase resistance in melanoma cells is associated with metabolic reprogramming, glucose dependence, and glutamine addiction. Mol Cancer Ther 12: 2581-2590, 2013.

25. Tsai WB, Aiba I, Long Y, Lin HK, Feun L, Savaraj N and Kuo MT: Activation of Ras/PI3K/ERK pathway induces c-Myc stabilization to upregulate argininosuccinate synthetase, leading to arginine deiminase resistance in melanoma cells. Cancer Res 72: 2622-2633, 2012.

26. Liu J, Ma J, Wu Z, Li W, Zhang D, Han L, Wang F, Reindl KM, Wu E and Ma Q: Arginine deiminase augments the chemosensitivity of argininosuccinate synthetase-deficient pancreatic cancer cells to gemcitabine via inhibition of $N F-\kappa B$ signaling. BMC Cancer 14: 686, 2014.

27. Walts AE, Bomalaski JS, Ines D and Orsulic S: Argininosuccinate synthetase (ASS) deficiency in high-grade pulmonary neuroendocrine carcinoma: An opportunity for personalized targeted therapy. J Cancer Res Clin Oncol 141: 1363-1369, 2015.

28. Horie S, Endo K, Kawasaki H and Terada T: Overexpression of MDM2 protein in intrahepatic cholangiocarcinoma: Relationship with p53 overexpression, Ki-67 labeling, and clinicopathological features. Virchows Arch 437: 25-30, 2000.

29. Zhao W, Zhang B, Guo X, Zhang X, Hu J, Hu X and Lu Y: Expression of Ki-67, Bax and p73 in patients with hilar cholangiocarcinoma. Cancer Biomark 14: 197-202, 2014.

30. Iguchi $T$, Yamashita N, Aishima S, Kuroda $Y$, Terashi $T$, Sugimachi K, Taguchi K, Taketomi A, Maehara Y and Tsuneyoshi M: A comprehensive analysis of immunohistochemical studies in intrahepatic cholangiocarcinoma using the survival tree model. Oncology 76: 293-300, 2009.
31. Schiffman SC, Nowacki MR, Spencer L, McMasters KM, Scoggins CR and Martin RC: Molecular factors associated with recurrence and survival following hepatectomy in patients with intrahepatic cholangiocarcinoma: A guide to adjuvant clinical trials. J Surg Oncol 109: 98-103, 2014.

32. Tan GS, Lim KH, Tan HT, Khoo ML, Tan SH, Toh HC and Ching Ming Chung M: Novel proteomic biomarker panel for prediction of aggressive metastatic hepatocellular carcinoma relapse in surgically resectable patients. J Proteome Res 13: 4833-4846, 2014.

33. Yang H, Lin M, Xiong FX, Yang Y, Nie X and Zhou RL: Reduced expression of ASS is closely related to clinicopathological features and post-resectional survival of hepatocellular carcinoma. Oncol Lett 1: 31-36, 2010.

34. Yoon CY, Shim YJ, Kim EH, Lee JH, Won NH, Kim JH, Park IS, Yoon DK and Min BH: Renal cell carcinoma does not express argininosuccinate synthetase and is highly sensitive to arginine deprivation via arginine deiminase. Int J Cancer 120: 897-905, 2007.

35. You M, Savaraj N, Wangpaichitr M, Wu C, Kuo MT, Varona-Santos J, Nguyen DM and Feun L: The combination of ADI-PEG20 and TRAIL effectively increases cell death in melanoma cell lines. Biochem Biophys Res Commun 394: 760-766, 2010.

36. Feun L, You M, Wu CJ, Kuo MT, Wangpaichitr M, Spector S and Savaraj N: Arginine deprivation as a targeted therapy for cancer. Curr Pharm Des 14: 1049-1057, 2008.

37. Wangpaichitr M, Wu C, Bigford G, Theodoropoulos G, You M, Li YY, Verona-Santos J, Feun LG, Nguyen DM and Savaraj N: Combination of arginine deprivation with TRAIL treatment as a targeted-therapy for mesothelioma. Anticancer Res 34: 6991-6999, 2014.

This work is licensed under a Creative Commons Attribution-NonCommercial-NoDerivatives 4.0 International (CC BY-NC-ND 4.0) License. 